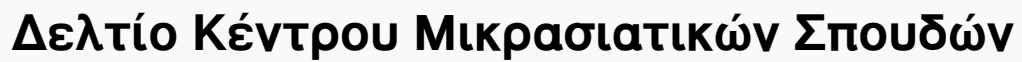

Tóp. 7 (1988)

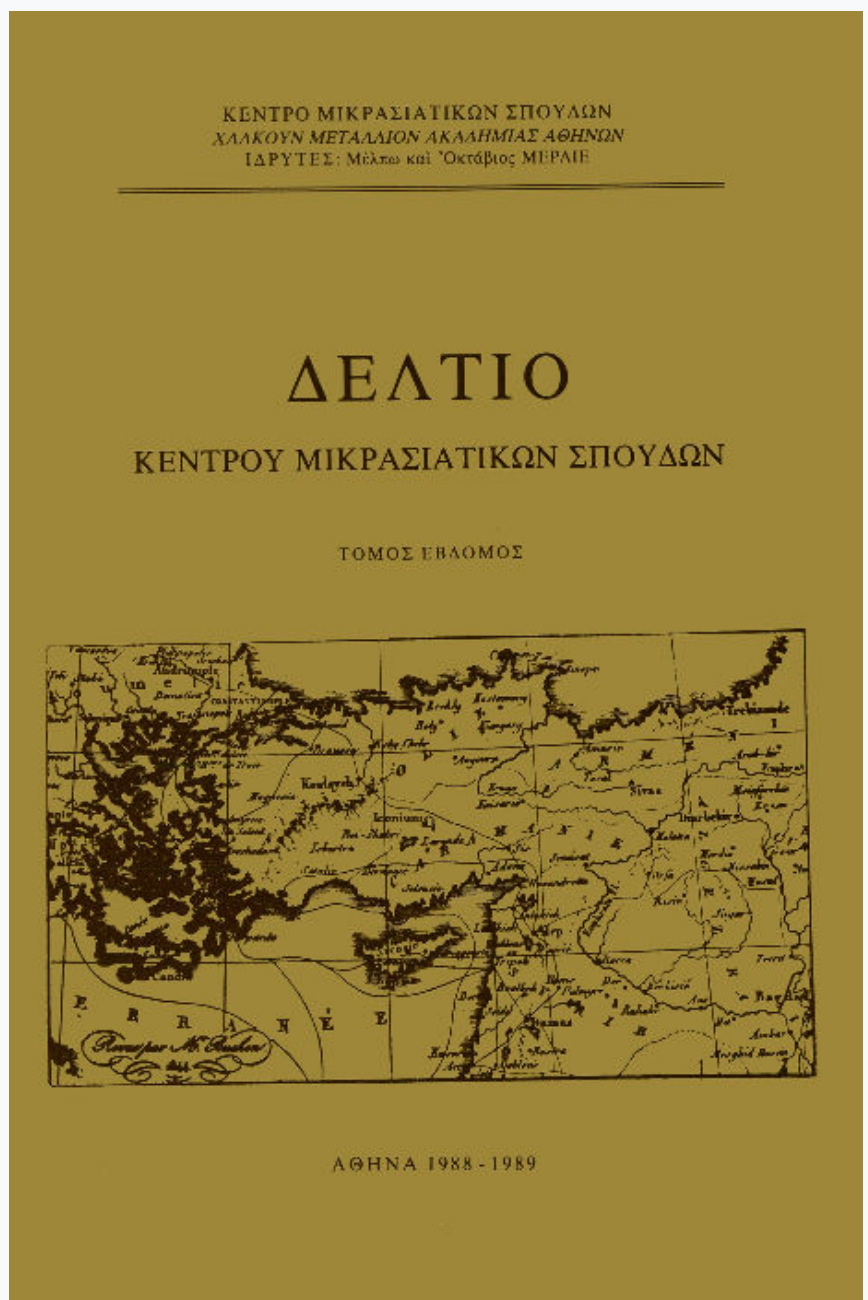

\section{La deterioration des sites et monuments de Cappadoce}

\section{Nicole Thierry}

doi: $10.12681 /$ deltiokms.202

\section{Copyright $@ 2015$, Nicole Thierry}

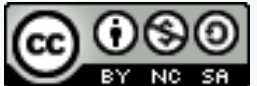

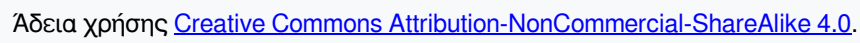

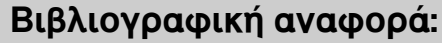

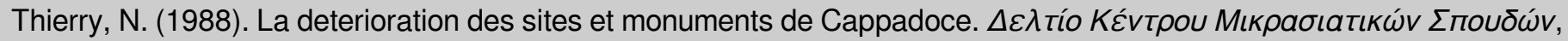
7, 335-354. https://doi.org/10.12681/deltiokms.202 
XPONIKA 



\section{NICOLE THIERRY}

\section{LA DÉTÉRIORATION DES SITES ET MONUMENTS DE CAPPADOCE}

\section{Introduction}

Notre époque est celle des bilans, et notamment des patrimoines archéologiques. Les changements de conditions économiques et sociopolitiques ainsi que l'altération rapide de l'écologie mondiale amènent les diverses communautés internationales à prendre la mesure de leurs responsabilités envers les témoins du passé. C'est l'heure des inventaires et des prévisions financières. Comme en médecine, la prévention sera moins coûteuse que les soins réparateurs, aussi ne faut-il pas craindre d'établir rapidement les listes de monuments à conserver. Pour notre part, une longue pratique de la Turquie nous permet quelques remarques sur les monuments chrétiens que nous y avons étudiés.

Nous sommes tout à fait conscients du nombre et de l'importance des sites archéologiques de tous types à sauvegarder sur le vaste territoire de la Turquie. Et, pour ce qui concerne les monuments byzantins, nous n'ignorons pas les travaux déjà faits dans quelques églises de Cappadoce et ailleurs, à Xanthos, Alahan Manastiri, Ephèse, etc. ${ }^{1}$. Cependant, nous pensons qu'il n'est pas inutile d'analyser l'état des conditions de conservation des sites et monuments ${ }^{2}$.

Si l'on compare l'inventaire actuel des monuments de Cappadoce avec celui des débuts du siècle, on observe d'une part, un réel enrichissement qui découle

1. Ces faits ont été précisés par M. Bülent AKARCALI, député et Co-Président de la commission Parlementaire mixte Turquie-CEE, dans une note de quatre pages communiquée au Parlement européen à Strasbourg le 17 janvier 1989.

2. Nous avons présenté un court rapport sur l'état des monuments byzantins et géorgiens de Turquie dans un colloque organisé au Parlement européen de Strasbourg le 17 janvier 1986. Ce colloque se déroulait dans une salle voisine de celle où se tenait la commission turco-européenne et nous avons pu secondairement parler de ces faits avec M. Bülent AKARCALI qui est tombé d'accord sur la nécessité de la conservation de ces monuments. 
des progrès de l'investigation archéologique ${ }^{3}$, d'autre part un appauvrissement qui provient de dégradations de plusieurs types. Mais à leur tour les monuments nouvellement découverts sont sujets aux destructions. Il convient donc de faire une analyse des processus de déterioration.

Les causes de destruction sont naturelles et humaines, ces dernières pouvant être actives ou plus simplement passives. Un caractère particulier du devenir du patrimoine archéologique de la Turquie vient de ce que le sort des monuments est souvent laissé aux soins des municipalités. Or, la notion de «monument historique» comme mémoire du passé n'apparait pas clairement aux autorités locales, et elles ne voient pas la nécessité d'entretenir un édifice qui n'a aucun usage. Ainsi avons nous vu souvent des églises conservées parce qu'elles étaient transformées en mosquées, en garages, ou en greniers, alors que celles qui étaient à distance des agglomérations étaient utilisées comme carrières de pierres. On peut espérer que le tourisme attirera l'attention des populations sur l'attrait qu'inspirent les vieux monuments. Nous verrons cependant que le tourisme sous la forme "tourisme de masse» devient à son tour un important facteur de destruction ${ }^{4}$.

Les monuments chrétiens ne sont pas seuls en cause, des monuments musulmans ont également souffert ou sont menacés ${ }^{5}$. Bien des destructions sont la conséquence du développement des agglomérations, en raison de la poussée démographique. Dans les villes, les monuments anciens sont rasés et remplacés par des immeubles de rapport ${ }^{6}$. Dans les villages, les pierres sont récupérées pour de nouveaux édifices, écoles ou mosquées ${ }^{7}$. L'exemple des destructions déjà anciennes d'une grande partie des églises de Bin Bir kilise, dans le Karadağı est bien connu ${ }^{8}$; dans le village actuel, le visiteur remarque aisément les

3. Ainsi, le nombre des peintures rupestres répertoriées en Cappadoce a plus que doublé depuis G. de JERPHANION (1942).

4. Nous avons également évoqué ces problèmes au Symposium européen de Bologne, «Science, Technology and European Cultural Heritage» (13-16 juin 1989); résumé à paraitre dans les Proceedings.

5. Un barrage prévu sur le Tigre doit détruire l'admirable site d'Hisn Kayfa (décrit par GABRIEL, p. 55-82, fig. 44-67).

6. Des maisons traditionnelles ont été abattues par rues entières, alors qu'elles auraient pu être réhabilitées suivant les techniques actuelles. En Cappadoce, citons la disparition de plusieurs quartiers du vieux Kayseri, et actuellement en cours celle des belles maisons de pierre de la petite ville d'Incesu.

7. Ainsi a été détruit dans les années 1980 l'ensemble monastique d'Opiza, situé près d'Artvin, à l'est d'Erzurum, et datant des $\mathrm{IX}^{\mathrm{e}}$ et $\mathrm{X}^{\mathrm{e}}$ siècles. Le fait est isolé dans cette région où la plupart des muhtars de villages prennent soin de leurs monuments (actuellement, le Directeur de la Culture et du Tourisme d'Artvin s'efforce de faire respecter un plan de conservation des beaux monuments géorgiens voisins)

8. Depuis les gravures de L. de LABORDE (1826), la description de Miss BELL et W. 
nombreux réemplois. Le fait se voit ailleurs, en Syrie du nord, par exemple dans la région dite "des villes mortes", des Ve-VIe siècle.

En Turquie, la recherche d'éventuels trésors est également à l'origine d'écroulement des monuments ou constitue une menace pour leur conservation. En effet, des trous profonds sont parfois creusés au pied des fondations, comme on peut le voir dans la Kızıl kilise de Sivrihisar, dans le Hasan dağı.

Nous envisagerons successivement les monuments de Cappadoce construits et rupestres. Les facteurs de détérioration étant différents suivant les cas.

Les édifices sont plus souvent détruits que les monuments rupestres car on réutilise leurs pierres. C'est le cas récent de la basilique d'Eski Andaval, près de Nigde. Du V ${ }^{\mathrm{e}}$ siècle, elle s'était conservée jusqu'en 1983, date à laquelle elle servit de carrière pour l'édification voisine d'une maison. Les travaux ont été interrompus en 1985, puis le site a été nettoyé et les ruines restantes consolidées en 1988-89. Ainsi, les pouvoirs publics se sont alarmés et ont mis fin à une désolante initiative locale. Cependant, l'intérieur éventré présente des peintures du $\mathrm{XI}^{\mathrm{e}}$ siècle que les intempéries risquent de faire disparaitre ${ }^{9}$.

D'autres églises paléochrétiennes situées au sud de Kayseri n'ont pas eu cette dernière chance. Depuis le début du siècle, trois sur six ont disparu (Suvaza, Tomarza en 1923, Skupi en 1954) et une autre a été à demi-détruite vers 1970 pour faire un barrage devant une petite mare (Buzluk de Persek, fig. 1). En 1987, nous avons découvert une église appartenant à cette série dans le village de Hanköy, mais ceci ne compense pas cela ${ }^{10}$.

Au sud de Nevşehir, les églises des villages de Soğanlı et de Tilköy ont aussi été détruites pour prendre leurs pierres (à une date indéterminée après 1927) ${ }^{11}$.

D'autres monuments, en raison de leur isolement en montagne n'ont souffert que des intempéries ou des tremblements de terre; c'est le cas des églises de Göreme d'Argée ( $V^{e}$ et $\mathrm{VI}^{\mathrm{e}} \mathrm{s}$.) et Çanlı kilise de Çeltek $\left(\mathrm{XI}^{\mathrm{e}}\right)^{12}$.

RAMSAY (1909) et celle de S. EYICE (1971), on peut mesurer la disparition des monuments. Bibliographie détaillée dans S. EYICE, p. 2-12, 201-2.

9. ÖTÜKEN 1987. État en 1987 dans DOSSIERS-ARCHEOLOGIA nº 121, p. 24.

10. Pour ces églises, RESTLE 1979, carte et index. La dégradation de Persek est postérieure à la visite de l'auteur (nos photos sont de 1976). Pour Hanköy, voir dans la prochaine livraison des Monuments Piot.

11. RESTLE 1979 , p. 24-26, 30-33. La corniche de la première a été identifiée dans le mur d'une maison par Natalia Teteriatnikov (communication personnelle).

12. RAMSAY-BELL, p. 404-48; RESTLE 1979, p. 42-44, 53-55. 


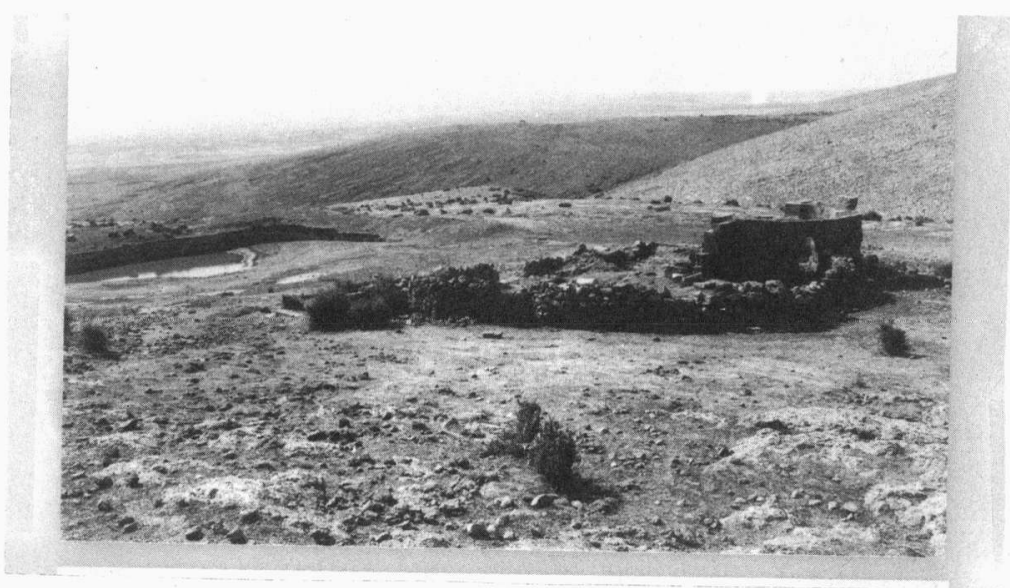

Fig. 1. L'église paléochrétienne Buzluk de Persek avec la retenue d'eau construite de ses pierres (état en 1976).

En Cappadoce rupestre, le problème de la conservation est plus complexe. Solidaires de la montagne, des falaises et des rochers, les églises troglodytes n'ont longtemps été détruites que par l'érosion. Depuis une bonne décennie s'est ajoutée la déprédation humaine due aux divers effets du tourisme de masse.

Les causes naturelles de destruction sont les effets de l'érosion. Elles ont fait l'objet de recherches privées et officielles, donné lieu à des prévisions et dans le cirque de Göreme, entrainé des mesures ponctuelles de protection ${ }^{13}$.

En Anatolie, l'érosion du vent, de l'eau et du gel, est intense. Les falaises s'effondrent, emportant les églises, en partie, voire en totalité.

L'érosion est variable suivant la dureté du rocher, ainsi voit-on des destructions stratifiées, comme à Yamanlı kilise, près d'Avanos, où le plafond seul

13. GÖREME HISTORICAL NATIONAL PARK, Master Plan 1972, p. 11, 24; U. Andolfato et F. Zucchi dans ARTS DE CAPPADOCE, p. 59-62; P. M. Schwartzbaum, dans le cadre de la structure d'association UNESCO-ICCROM-Ministère turc de la Culture et du Tourisme (direction des Antiquités et des Musées), p. 52-57, dans EPSTEIN 1986. 
a été détruit, l'église paraissant comme décapitée ${ }^{14}$. Ailleurs, des glissements de terrain envahissent les cavités, comme dans la basilique enterrée de MaçanAvcilar ${ }^{15}$.

Le phénomène est en pleine évolution dans le vallon d'Ihlara où, sur la rive gauche de la rivière, les entrées ou les absides des églises ont disparu, suivant la direction des méandres. Récemment, le porche de Pürenli Seki kilise s'est écroulé, alors qu'en face, l'entrée de la Bezirhanı kilisesi a été obturée par un chaos $^{16}$. A Çavuşin, près de Göreme, depuis 1964 plusieurs effondrements de la falaise ont détruit une partie du village et la dernière colonne extérieure de la basilique du $\mathrm{V}^{\mathrm{e}}-\mathrm{VI}^{\mathrm{e}}$ siècle est tombée en 1975 . La perte de ce dernier élément du portique à l'antique qui précédait la superbe façade est très regrettable pour l'archéologue comme pour l'amateur d'art.

Pour le devenir des peintures, le plus grave danger est celui des infiltrations d'eau qui délitent la roche et décolle l'enduit. Le processus a été défini à propos de Tokal ${ }^{17}$; cette érosion est très active dans certains vallons des environs de Göreme, menaçant notamment l'intégrité de Kilıclar kilise et du Pigeonnier (nommé aujourd'hui Mereymana), ayant fait récemment disparaitre l'église $\mathrm{n}^{\circ}$ $6 a^{18}$. Tous les ans l'inventaire se solde par de nouvelles dégradations; ainsi avons-nous constaté cet été 89 que dans l'église d'Hagios Basilios, la célèbre croix de la paroi sud est tombée à moitié ${ }^{19}$. Cependant, le processus est très différent d'une église à une autre, et certaines n'en ont pas souffert depuis près de trente ans, dans le vallon du Güllü dere par exemple.

La lutte contre la dynamique érosive est evidemment très difficile, celle-ci s'étant accélérée depuis peu par le creusement de nouveaux établissements rupestres, l'ouverture de routes et l'établissement de vastes parkings. Les mesures conservatoires sont empririques et aléatoires; le drainage efficace des eaux de ruissellement nécessiterait sans doute une étude globale des bassins hydrographiques.

$$
\text { 它 }
$$

Les causes humaines d'altération des sites et des monuments sont d'apparition récente. Le Père de Jerphanion, lors de son dernier voyage en 1927, avait

14. N. THIERRY, dans DOSSIERS-ARCHEOLOGIA n ${ }^{\circ} 121$, p. 37.

15. DOSSIERS-ARCHEOLOGIA ${ }^{\circ} 121$, p. 22, 23.

16. THIERRY, N. et M., 1963, carte p. 22.

17. P. M. SCHWARTZBAUM dans EPSTEIN 1986, p. 54.

18. Voir les fentes le long desquelles se font les ruptures dans RESTLE 1967, fig. 251-301 et $57-60$.

19. G. de JERPHANION, II, p. 107-08. 


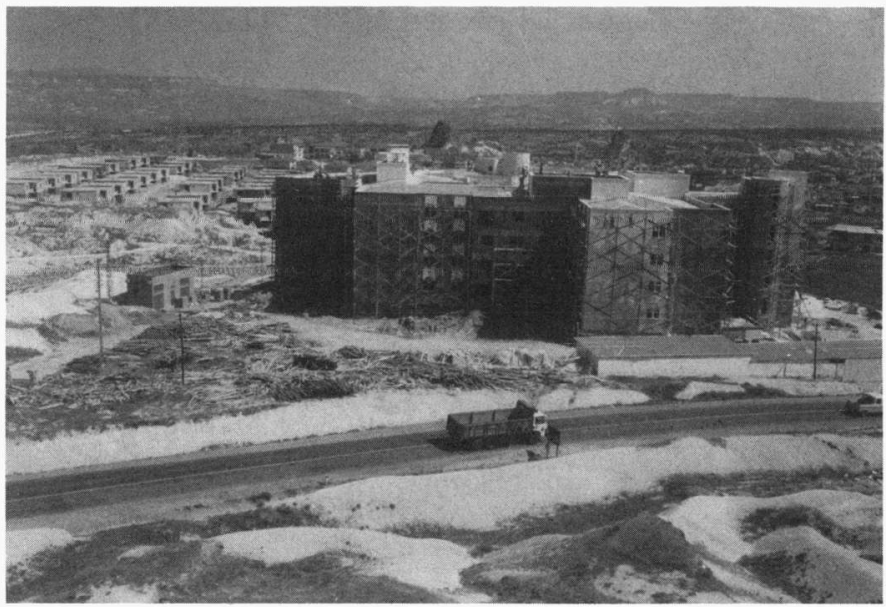

Fig. 2. Ortahisar. L'hotel Lapis et ses pavillons, en construction (1987).

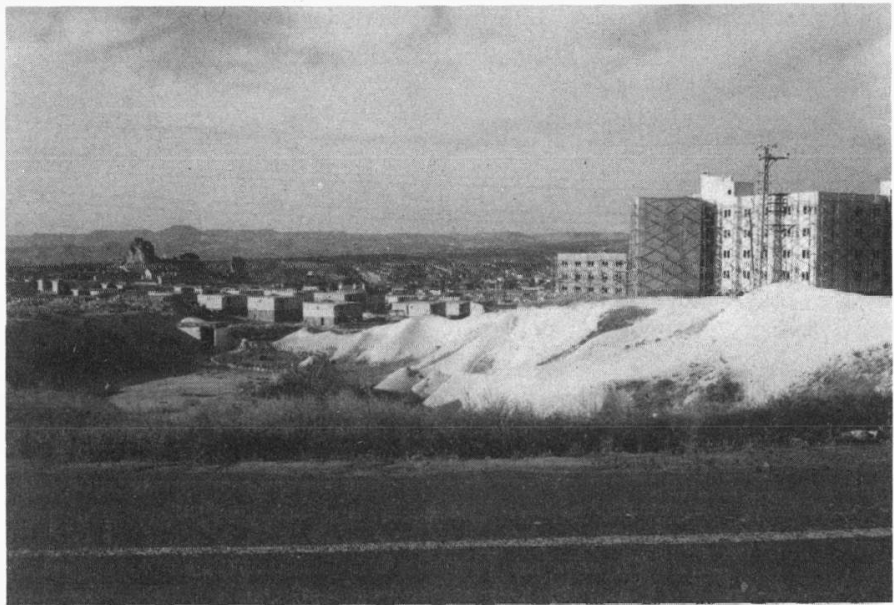

Fig. 3. Ortahisar. L'hotel Lapis et ses pavillons dans le paysage (1988). 
constaté d'importantes déprédations consécutives à l'abandon de la région par la population grecque; il en tirait des prévisions pessimistes qui ne se vérifièrent pas dans l'immédiat ${ }^{20}$. En effet, les monuments tombèrent dans l'abandon et leur état se modifia assez peu entre sa visite et la dernière décennie.

Par contre, les conditions ont changé depuis quelques années, car pour son malheur la Cappadoce rupestre est devenu un vaste champ touristique, et la région d'Ürgüp-Nevşehir un des plus importants lieux d'appel pour l'industrie hôtelière et pour la vente des tapis et produits artisanaux. Malgré les plans de protection des sites et des monuments ${ }^{21}$ les installations sont anarchiques pour la plupart. Enfin, le très grand nombre de visiteurs est par lui-même source de nuisances directes et indirectes.

Nos exemples sont essentiellement localisés dans la region d'Ürgüp où s'est développé un tourisme de masse sans rapport avec les capacités de l'environnement naturel.

\section{Dégradation des sites, des agglomérations et de l'environnement}

D’année en année on assiste à la destruction des beaux ensembles de Göreme, Maçan-Avcilar, Avanos, Ürgüp, etc., et le phénomène va s'accélérant.

En premier lieu, il y a une vingtaine d'années, de nouveaux villages ou de nouveaux quartiers ont été hâtivement construits, inesthétiques et inadaptés à l'environnement, ainsi à Ortahisar et à Çavuşin. Dans les villages et les villes, les centres anciens furent ravagés pour divers aménagements; comme plus loin à Kayseri et Incesu, ce fût le cas ici à Ürgüp et Avcilar (récemment rebaptisé Göreme). Ces faits étaient en partie la conséquence de l'expansion démographique; ils relevaient d'une loi générale et l'on pouvait seulement regretter de les observer également en Cappadoce.

Il est plus surprenant de constater que dans les sites réservés aux touristes, on a développé un équipement particulièrement aggressif, depuis les panneaux publicitaires les plus grands possible jusqu'aux hotels géants. À la sortie du village d'Ortahisar, jadis pittoresquement serré aux pieds de son haut rocher, on a construit un énorme hotel et un village de vacances; les cinq étages de la bâtisse écrasent le paysage qu'enlaidissent encore les pavillons attenants semblables à ceux d'une cité ouvrière (fig. 2 et 3 ) $^{22}$. Non loin, dans le site du Parc

20. G. de JERPHANION, p. v-vi de la seconde partie du t. I. Ses premiers inventaires datent de $1907,1911,1912$.

21. Celui de 1967 a été révisé et publié en 1972 (GÖREME HISTORICAL NATIONAL PARK, Master Plan). Un projet turc de "Conservation structurelle de Göreme» a commencé en 1982. Le site a été inscrit sur la liste du Patrimoine mondial de l'UNESCO en 1985. Les églises de Tokalı, Elmalı et Karanlık ont été l'objet de restaurations, de 1973 à aujourd'hui. .

22. Cet Hotel Lapis n'est pas conforme au Plan de la région qui limite les élévations à trois 


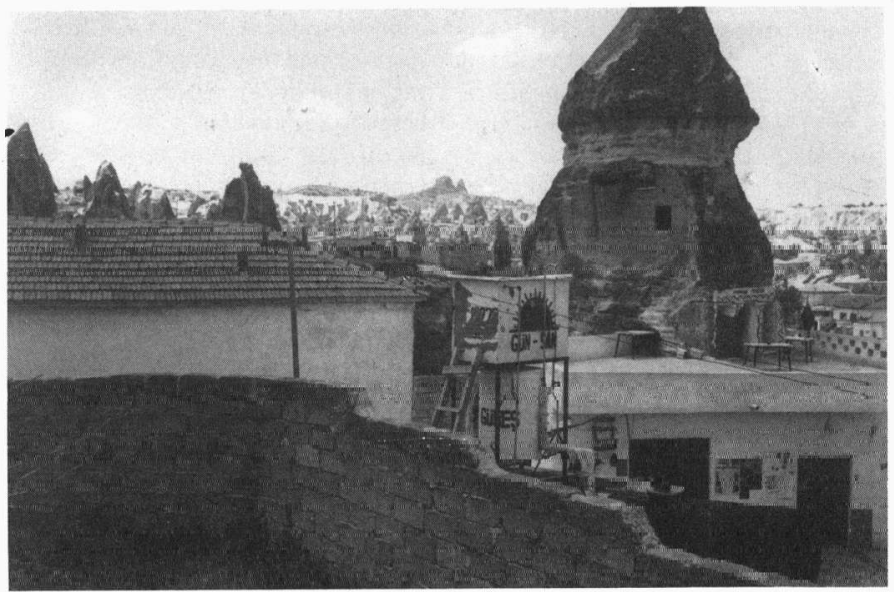

Fig. 4. Tombeau romain utilisé comme bar d'une lokanta à l'entrée de Maçan-Avcılar (état en 1988).

national de Göreme, on a édifié un vaste hotel à l'entrée du vallon d'En Nazar, un des lieux les plus pittoresques de la région; pour l'établir sous la longue crête de Maçan-Avcılar, on a arasé une série de cônes. Là d'ailleurs, à l'entrée de Maçan, l'harmonieux ensemble des vignes et des cônes a été détruit par l'installation de motels, campings et restaurants. L'un de ces derniers étale ses réservoirs, murs de ciments et annexes de bidonville; son bar en terrasse est un ancien tombeau romain défiguré (fig. 4) (23 $^{23}$ De l'autre côté du village, sous une ancienne maison grecque transformée en restaurant de luxe, le cirque naturel est aujourd'hui en voie de recreusement pour l'édification d'un amphithéatre

étages; il a été l'objet de nombreuses critiques (cf. l'article dans Hürriyet du 16-11-1988). Contrairement à celui-ci, quelques hotels de la région sont d'assez belles réalisations architecturales. La plupart se groupent dans les villes ou leurs environs immédiats (Ürgüp, Avanos et Nevșehir), ce qui devrait être la règle pour préserver les paysages. L'hotel déjà ancien du Club Méditerranée, à Uçhisar, est un des rares exemples de discrétion architecturale et d'adaptation à son environnement.

23. En 1982 nous avions déjà publié les divers états antérieurs de ce tombeau (DOSSIERS ARCHEOLOGIA $n^{\circ} 63$, p. 23). 
destiné à des spectacles touristiques(?). Les propriétaires du lieu ont également détruit en partie l'église du Haut Moyen Age qui constituait les caves $^{24}$.

Les besoins touristiques ont attiré toute une nouvelle population, outre les hotels, restaurants, boites de nuit, emporiums, ateliers de tissages et poteries, fabriques d'objets d'onyx, sont nés des commerces plus ordinaires, des établissements publics, postes, écoles et mosquées. De Maçan-Avcilar à Avanos, les constructions de tous ordres gagnent le long des routes qu'on a multipliées et la campagne est transformée en longue agglomération. Le milieu rural est modifié, les maisons deviennent pensions, les jardins campings. Les paysages sont remaniés et l'on détruit rochers, nature sauvage et cultures.

Plus loin dans la campagne des «point\$-de-vue» panoramiques sont cimentés et pourvus de parkings et d'éventaires et des sites charmañts sont saccagés comme celui de l'ermitage de Siméon, près de Zelve. Des routes transversales mènent à de nombreux sites qu'on atteignait naguère après d'agréables promenádes pédestres; bientôt ces routes de terre seront asphaltées pour que les touristes arrivent par autocars entiers. C'est déjà le cas de la route de crête qui mène d'Ortahisar au pied du Aktepe, au-dessus de l'Église du stylite Nicétas à laquelle on descend par un court sentier fléché.

Quant au cirque naturel du Musée de Göreme, il a été remodelé pour rendre son accès plus aisé aux bus; les bulldozers ont élargi la route et nivelé un vaste parking. Celui-ci cependant reste encore insuffisant pour les périodes de grande affluence; ainsi a-t-on pu compter cinquante-deux autocars un jour de mai 1989, nombre qui n'est pas un record et qui est bien dépassé en août.

Dans les vallons autour d'Ürgüp et d'Ortahisar, on a tracé de nombreux chemins piétonniers destinés à mener aux églises tout en endiguant le flot des touristes. Ils constituent cependant une nuisance pour les paysans, car les promeneurs s'écartent volontiers et leur piétinement à travers champs et vergers abime les cultures, le dommage étant souvent aggravé par la cueillette indélicate des fruits et grappes de raisins.

Si l'on ajoute que certaines églises dans lesquelles s'introduisent les visiteurs sont des granges encore en usage, des celliers, voire des pièces d'habitations, et que, d'autre part, les autorités locales interdisent aux villageois toute fouille de cavités fortuitement découvertes, on comprend que ceux-ci se sentent lésés par le nouveau destin de la région. En fait, les intérêts des agriculteurs s'opposent souvent à ceux des exploitants du tourisme (guides, commerçants, hôteliers). Ainsi s'explique en partie l'hostilité que manifestent certains paysans, en opposition avec leur ancienne tradition de gentillesse et d'hospitalité.

24. Il s'agit de «'Église sous les tombeaux», en face du quartier dit Karşı becak, là où sont les plus anciens établissements de Maçan. 


\section{Dégradation des monuments et des peintures}

Les causes de destruction des monuments rupestres sont multiples.

Avant l'ère du tourisme, les dégradations étaient surtout celles du temps et accessoirement celle d'un paysan soucieux d'aménager son bien. Ainsi trouvet-on des pièces annexes élargissant des tombeaux antiques (comme à Maçan) ou des chapelles (comme dans l'Église de Nicétas), et des cuves à raisin recreusées dans les parois et les sols (comme dans l'Église $\mathrm{n}^{\circ} 2$ de Zelve). La transformation en pigeonnier avait comme inconvénient le creusement de trous pour fixer les perches et de cuvettes pour la nidation; elle eut l'avantage de maintenir les peintures dans l'obscurité, ce qui assura la protection des pigments. Ainsi s'explique l'éclat actuel des couleurs dans le Kuşluk de Kızıl Çukur.

Cependant, c'est pour nettoyer son pigeonnier que vers 1960, un villageois de Soğanlı arracha les peintures de Ballı kilise; il ne reste rien de cet intéressant décor du $\mathrm{X}^{\mathrm{e}}$ siècle $^{25}$.

$$
\text { is }
$$

Depuis le développement du tourisme de masse, les dégradations se sont multipliées et sont de plusieurs ordres.

Dans les sites trop visités de Soğanlı et surtout de Göreme les peintures murales sont rongées par l'acide carbonique et la vapeur d'eau que rejette la respiration de milliers de visiteurs (jusqu'à 5000 par jour). De plus, dans ces églises relativement petites, la rotation des groupes est insuffisante pour qu'on admette chaque fois un petit nombre de visiteurs, si bien que ceux-ci, serrés contre les parois, frottent les peintures et les détruisent à la longue ${ }^{26}$.

D'autre part, dans certains monuments, pour faciliter la visite, on a élargi les entrées et même abattu des parois. Le cas le plus regrettable est celui de Karabaș kilise, à Soğanlı, où l'on a ouvert la nef funéraire en détruisant deux portraits de moines ${ }^{27}$. Ailleurs, les dégradations sont l'oeuvre de tour-operators sans scrupule, comme à Hallaç Manastir près d'Ortahisar ${ }^{28}$, où l'on organisait

25. G. de JERPHANION, II, p. 249-72. Nous les avons encore vues en 1955 mais n'en avons pris que deux photographies en couleurs.

26. Le plan de protection limitait les groupes à douze personnes et prescrivait une surveillance sévère par les gardiens (GÖREME HISTORICAL PARK, p. 25).

27. Une première destruction date des années 1960, complétée entre 1980 et 1982 . Description antérieure dans G. de JERPHANION; II, p. 356-57, et actuelle dans RODLEY 1985, p. 195.

28. RODLEY 1985, p. 11-25. 
des soirées dans les salles capitulaires, en fixant des flambeaux dans les colonnes.

Dans les églises isolées ou mal gardées, les peintures sont victimes à la fois des touristes indélicats et des musulmans fanatiques. Les touristes couvrent les parois de graffiti comme jadis les pélerins mais ces marques sont ressenties aujourd'hui comme une dégradation d'oeuvre d'art. Ce type de vandalisme est particulièrement développé dans le vallon d'Ihlara qui est un lieu de pique-nique très apprécié des touristes turcs; en vingt ans, nous avons vu à peu près disparaitre les peintures du vestibule d'Yllanlı kilise (IX ${ }^{\mathrm{e}}-\mathrm{X}^{\mathrm{e}}$ siècle $)^{29}$. Dans la région de Göreme, les églises devenues accessibles par des sentiers aménagés commencent à en souffrir également. Cependant, un vandalisme beaucoup plus dangereux a fait son apparition dans ce centre.

En aval du Musée en plein air de Göreme, Saklı kilise (nommée encore église $\mathrm{n}^{\circ} 2 \mathrm{a}$ de Göreme), dont la grille reste malheureusement ouverte, a été l'objet de plusieurs types de détériorations. Outre les graffiti de type banal nous avons constaté d'autres dégâts; dans les années 1986-87, de profondes entailles ont détruit les visages des évêques peints dans les absides, et en 1989 une série de taches brunes maculaient le visage et le torse du Christ en croix (fig. 5) ${ }^{30}$.

Cette attaque systématique des visages (et parfois des inscriptions voisines) nous parait caractéristique d'un réveil de l'intégrisme musulman dans la région.

Notre opinion est basée sur d'autres exemples, dont le nombre va croissant. Les peintures de la basilique Saint-Jean-Baptiste de Çavuşin, qui datent du VII ${ }^{\mathrm{e}}$ siècle, ont été sauvagement arrachées et lapidées dans l'hiver 1983-1984; l'admirable visage de l'ange qu'encadraient les Hébreux dans la fournaise est détruit (fig. 6, 7 et 8), comme dans l'abside, les bustes de la lune et du soleil ainsi que la grande croix, comme sur la paroi nord, les scènes de la trahison du Christ et du Jugement de Pilate ${ }^{31}$. Dans un vallon voisin, ce qui restait des visages à l'arc triomphal et dans l'abside de St-Georges de Zindanönü a été vigoureusement gratté dans l'hiver 1987-1988 (têtes des prophètes, de l'archange et du séraphin) ${ }^{32}$. À Göreme encore (mais tous ces sites sont très proches, à $2 \mathrm{~km}$ environ les uns des autres), des visages accessibles de Kilıclar

29. THIERRY, N. et M., 1963, p. 91-93.

30. Voir les sujets intacts dans RESTLE 1967, fig. 31, 36, 37.

31. Dans cette église la rage de destruction est évidente; sur le panneau des Hébreux, les vandales se sont même adressés aux étrangers en écrivant NON (fig. 8). Pour l'état antérieur, THIERRY, N., 1983, p. 66, 76, pl. 28, 29, 31, 35.

32. Étude à paraitre dans notre volume II. 


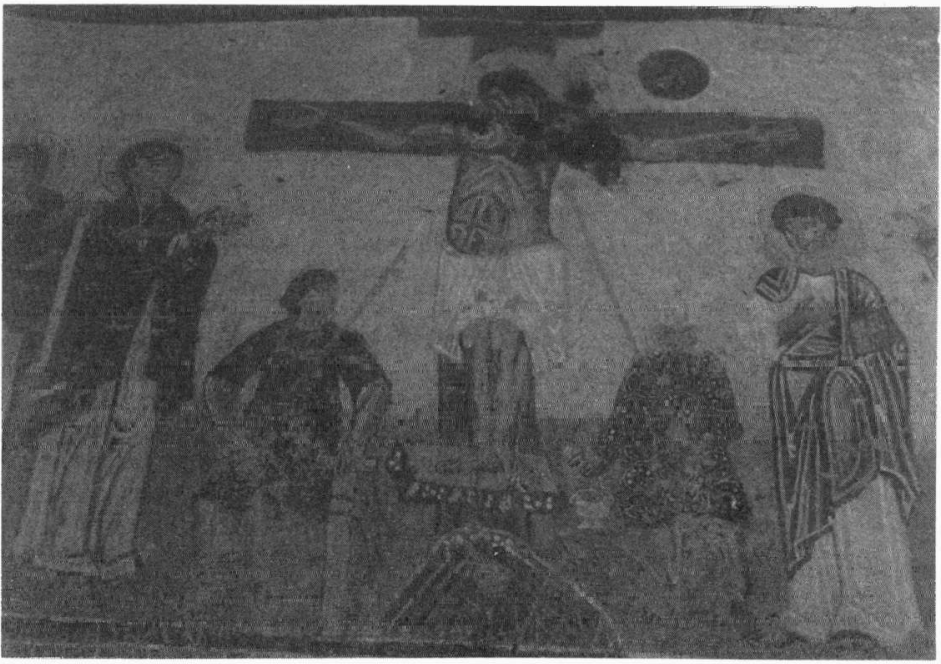

Fig. 5. Saklı kilise. Crucifixion (état en 1989).

kilise ( $\mathrm{X}^{\mathrm{e}}$ siẹccle) ont été récemment détruits. Il s'agissait des portraits des apôtres Jacques et Philippe figurant dans la scène de Pentecôte qui surmonte la porte d'entrée ${ }^{33}$; leurs visages et leurs noms ont été grattés jusqu'à disparition complète.

Cette série de dégradations malveillantes est centrée par le village de Çavuşin où le réveil de l'intégrisme dans une partie de la population est lié à une certaine hostilité aux touristes.

Des témoins nous ont également donné cette raison aux destructions récentes des figures absidales de l'église du village de Babayan ${ }^{34}$, de même pour. de nouvelles lapidations des visages dans l'Église des Quarante martyrs de Suveş, près du village de Şahinefendi ${ }^{35}$.

33. RESTLE 1967, fig. 275. 56).

34. Constatées en 1988 et récentes puisque postérieures à l'article de SCHIEMENZ 1986 (pl.

35. Pour l'état avant ces récents jets de pierres, RESTLE, fig. 414-32; les dommages ont surtout 


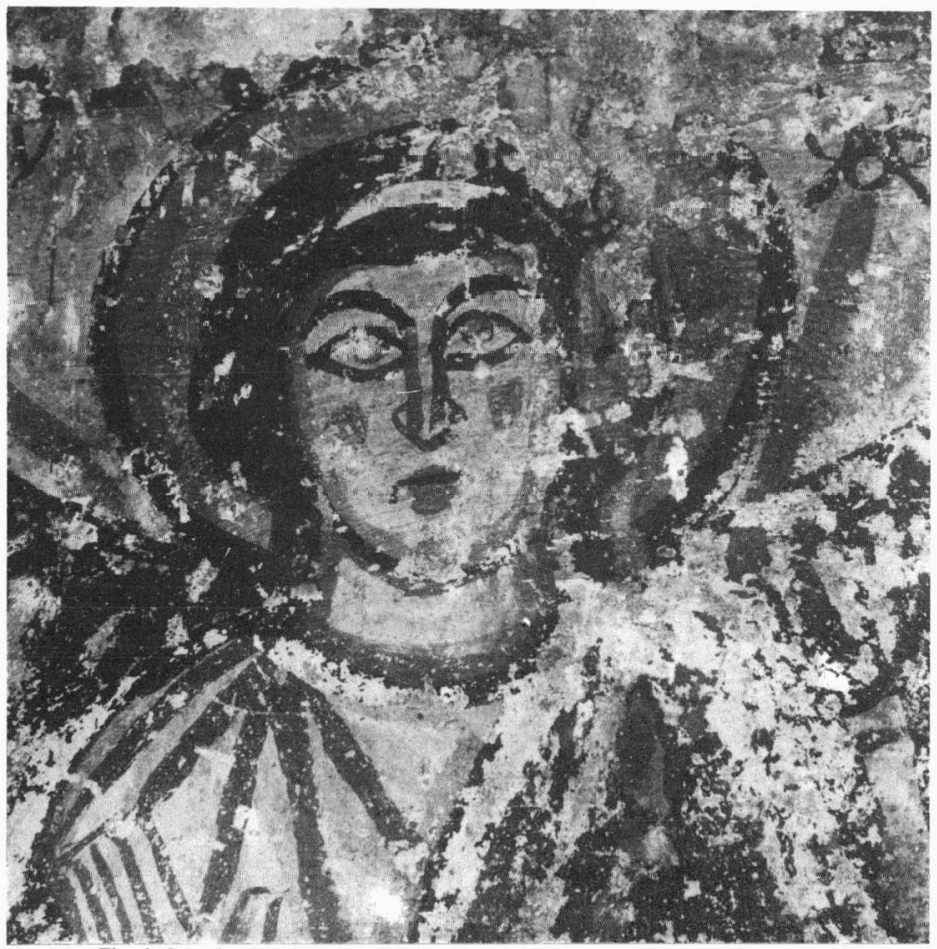

Fig. 6. Çavuşin. L'ange des trois Hébreux dans la fournaise (état en 1983).

À Soğanl, la destruction du dernier fragment de la Vision d'Eustache de Geyik kilisesi ${ }^{36}$ est plus ancienne et de motivation moins claire; quoi qu'il en soit, le beau cerf planté dans un paysage rocheux a été arraché dans les années 1970. Loin de la Cappadoce, au Latmos, nous avons constaté dans la Grotte du Pantocrator (VII-VIII ${ }^{\mathrm{e}}$ siècle), que la campagne photographique destinée à

affectés la scène du martyre des Quarante (la fig. 423, déjà en mauvais état n'est plus reconnaissable).

36. RESTLE 1967, fig. 467. 


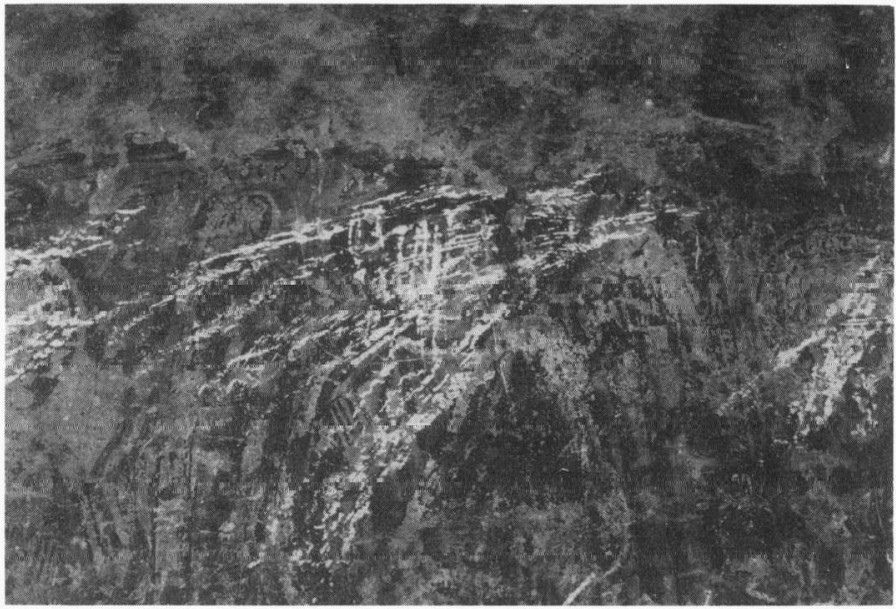

Fig. 7. Çavuşin. L'ange des trois Hébreux (état en 1984).

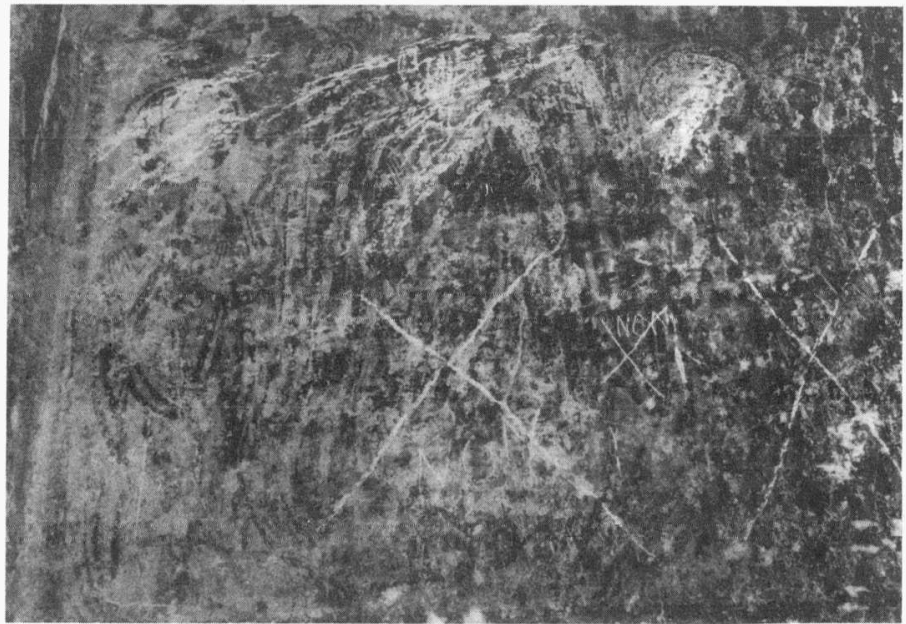

Fig. 8. Çavuşin. Destruction systématique des visages de la scène des trois Hébreux dans la fournaise (état en 1984). 
l'illustration de l'ouvrage de M. Restle avait attiré l'attention en ces lieux cependant déserts, puisque tout ce qui restait de visible, visages et protomes des évangélistes, a été arraché ${ }^{37}$.

Ces manifestations islamiques nous semblent dirigées à la fois contre les images du culte chrétien et contre les étrangers dont la présence constitue une offense à la morale musulmane et un risque de changement mauvais du milieu traditionnel. D'ailleurs, ce comportement est géographiquement très limité et ne s'observe habituellement pas dans les lieux où les touristes sont peu nombreux ou peu dérangeants. En ce sens, les agressions envers les peintures seraient en partie une réponse à l'agression du tourisme sauvage et une première prévention serait d'éduquer les deux groupes de population, celui des visiteurs et celui des habitants.

Citons enfin comme rare cause de destruction, la déposition de peintures ou sa tentative ${ }^{38}$. Les cas que nous connaissons sont anciens; ainsi avons-nous constaté en 1964 la disparition de trois beaux visages dans l'Église Saint-Jean de Güllü dere qui sert de pigeonnier (les peintures sont de 913-920); après un essai(?) sur une main dans la scène de la Dormition, on a découpé les têtes d'André et de Thaddée (fig. 9 et 10) et celles de la Vierge et de l'Enfant dans la scène de la Fuite en Égypte ${ }^{39}$. À Göreme, encore à Kilıclar kilise, dans les années 1970, on a tenté de déposer un sujet bien cadré, l'Annonce à Jean-Baptiste; le fragment encollé s'est mal détaché et les voleurs ont abandonné la peinture gravement endommagée ${ }^{40}$. En raison de l'aggravation des dégâts causés par à la fois par l'érosion et le vandalisme, les autorités locales ont fermé l'église; en 1989, la grille était close, un écriteau annonçant des restaurations futures. Espérons que la fermeture sera efficace et durable et les restaurations limitées à une consolidation, car là encore, le mot dégradation peut être employé à propos de restaurations.

37. RESTLE 1967, fig. 542, 543. Notre visite eut lieu en 1969. On verra (note suivante), que d'autres dégradations étaient le fait de pillards. Quant à la disparition des admirables peintures paléochrétiennes du tombeau hypogée des environs de Nicée, constatée en 1988 par des témoins dignes de foi, nous n'en connaissons pas la cause (ces peintures, découvertes intactes en 1967 et vite saqlpêtrées, ont été publiées par N. FIRATLI en 1974).

38. Le hasard nous a permis de constater la technique de voleurs de fresques au Latmos, en 1969; notre arrivée dans la grotte dite «de la Vie du Christ» les avait mis en fuite, abandonnant leur matériel, du plâtre prêt à recevoir les fragments arrachés; ceux-ci, répandus à terre en mille morceaux nous ont donné l'impression d'un saccage plutôt que d'une déposition efficace.

39. THIERRY, N., 1983, pl. 60, 80, 85. Pour les ensembles où ces têtes figuraient nous n'avons reproduit que des photos antérieures à 1964; mais on verra le détail de la main découpée dans un rectangle sur notre $\mathrm{pl} .71$. Que son devenus ces visages restés intacts jusque là?

40. RESTLE 1967, fig. 270. 


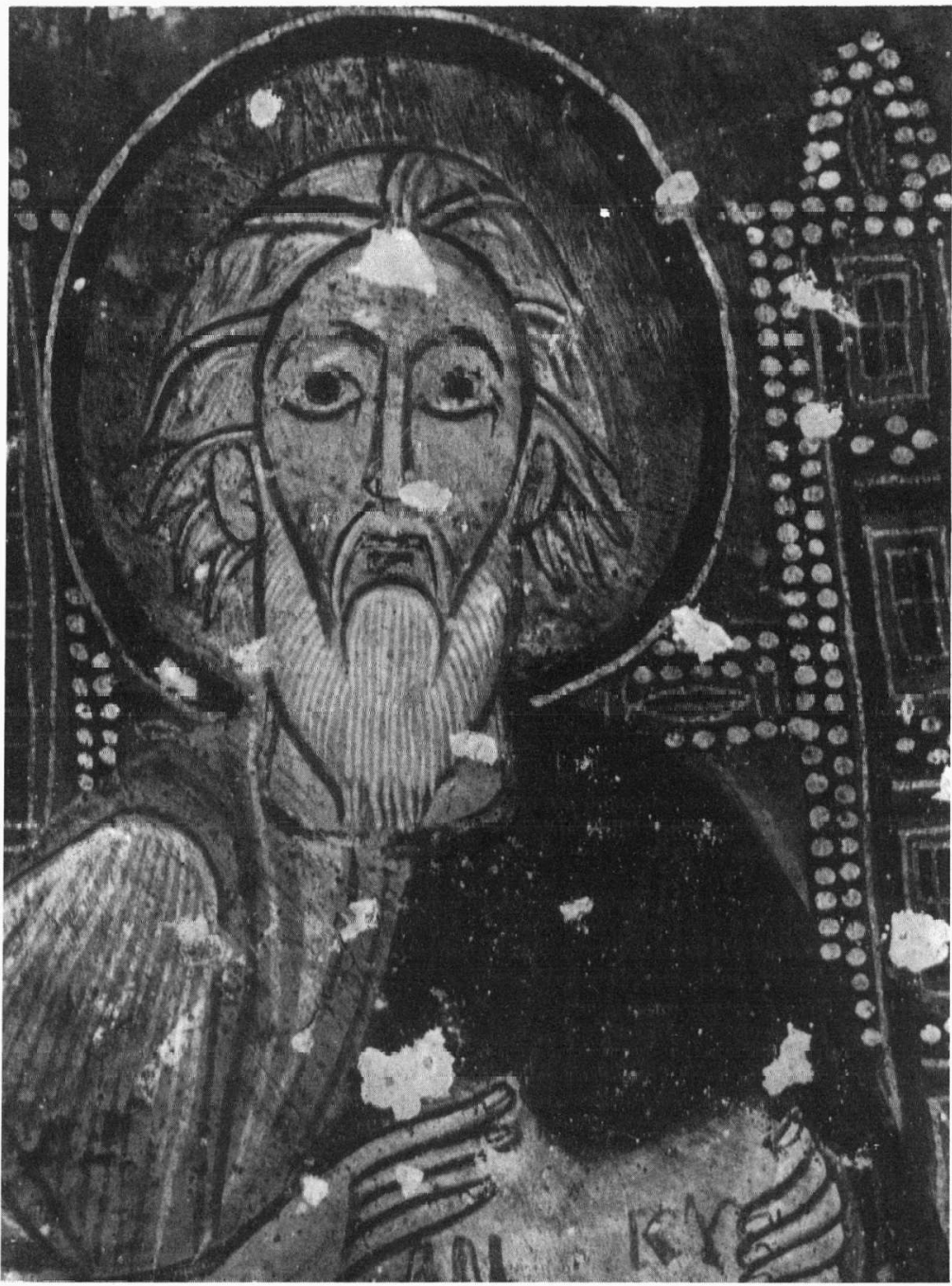

Fig. 9. St-Jean de Güllü dere. L'apôtre André (état en 1963). 
Dans la publication qui suivit les travaux entrepris dans l'Église de Tokalı à Göreme (1973-1980), l'accent a été mis sur les difficultés techniques de stabilisation des fissures et de fixation des peintures qui se détachaient du rocher. L'état précaire dû aux infiltrations d'eau et aux microorganismes et la friabilité du plâtre expliquent comment le nettoyage préalable entraina des pertes de substance importantes sur le bord des fragments en mauvais état ${ }^{41}$. D'autre part, contrairement à leur doctrine, les restaurateurs ont été amenés à compléter quelques sujets, notamment les scènes de l'Annonciation, de la Visitation et de l'Epreuve de l'eau; enfin, le visage du Christ de la bénédiction des apôtres a été refait. Les retouches ont altéré le style de ces morceaux ${ }^{42}$. Il faut cependant reconnaitre que ces travaux ont révélé d'admirables fragments de peintures cachés jusque là par la poussière et la fumée des bougies. Lorsque les décors étaient en bon état, les nettoyages ont, en effet, été tout à fait bénéfiques. C'est pourquoi on peut espérer de bons résultats à Karanlık kilise où les peintures étaient bien mieux conservées ${ }^{43}$.

Les avis seront toujours discutés en matière de politique de restauration, c'est pourquoi nous rappelons l'aspect satisfaisant des travaux entrepris par $\mathrm{M}$. Gough et son équipe à Eski-Gümüs, près de Nigde. Malgré un certain degré d'effacement des peintures qui étaient profondément encrassées, le style a été parfaitement conservé et nous pensons que ce résultat est dû à l'étroite collaboration entretenue entre l'équipe des techniciens et l'historien de l'art byzantin ${ }^{44}$.

41. P. M. SCHWARTZBAUM, Directeur en chef des restaurations pour l'ICCROM, dans EPSTEIN 1986, p. 52-57. Les illustrations ne rendent pas compte de ces manques particulièrement sensibles dans le couloir qui précède les absides, là où la série des portraits d'évêques a considérablement souffert (photographies personnelles avant et après les restaurations).

42. Ce jugement ne nous est pas particulier et reflète l'opinion de tous les amateurs éclairés. D'autre part, M. RESTLE 1986 (dont nous ne suivons pas la datation) a déjà exprimé les mêmes critiques, s'interrogeant sur le bilan de ces restaurations, "est-on obligé et est-on autorisé à conserver ou à restaurer?» (p. 351-52 et n. 15). Ajoutons cependant, que les retouches sont labiles car posées à l'aquarelle (communication épistolaire de M. Cevat Erder du 9-12-1986). D'autre part, la consolidation n'est garantie qu'une vingtaine d'années, en raison de la structure des résines synthétiques. Il est à craindre que la détérioration naturelle des peintures se poursuive compte tenu des conditions locales qui exigeraient une sévère protection de l'environnement et la limitation du nombre des visiteurs. Quoi qu'il en soit, on peut regretter que les admirables peintures de Tokalı aient servi d'entreprise-pilote, bien que P. M. SCHWARTZBAUM l'ai justifié par la situation d'urgence du monument (p. 54).

43. Il semble cependant que les techniciens ne travaillent guère avec des spécialistes des peintures byzantines.

44. GOUGH 1964 et 1965. Sur les difficultés rencontrés par les restaurateurs, cf. notre article sur d'autres exemples, en Arménie soviétique, THIERRY, N., 1981. 


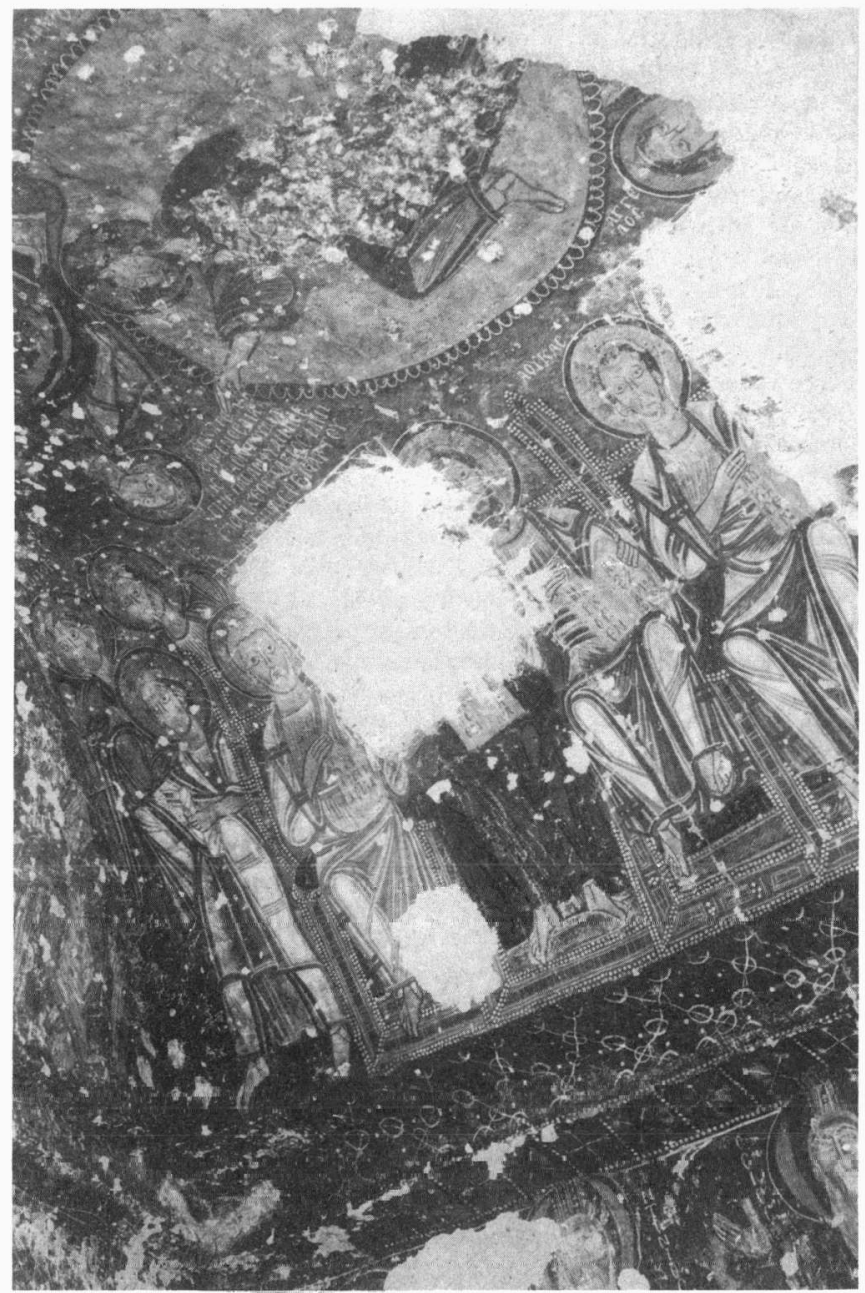

Fig. 10. St-Jean de Güllü dere. Emplacements vides de bustes d'André et Thaddée (état depuis 1964). 
En conclusion, l'avenir des sites et des églises rupestres de la Cappadoce de grand tourisme nous parait bien sombre. Tout concourt à leur destruction, même les publications savantes qui attirent l'attention des amateurs d'art puis celle des organisations touristiques. Les autorités officielles turques et internationales qui édictent des préceptes de conservation sont vite dépassées par le développement anarchique de l'industrie des loisirs. L'afflux des visiteurs détruit l'environnement et altère les traditions villageoises. Les monuments sont endommagés à la fois par la masse des visiteurs, le sans-gêne de certains d'entre-eux et l'hostilité induite chez les paysans musulmans.

Nous n'avons pas qualité pour proposer des remèdes qui auraient eux-aussi leurs inconvénients. Nous ne pouvons que constater le manque d'un plan d'exploitation touristique respectueux de l'écologie locale et des monuments ${ }^{45}$, et surtout, le manque d'une volonté d'application rigoureuse d'un tel plan qu'il aurait fallu imposer aussi bien aux grdupes financiers qui ont investi dans la région qu'aux municipalités qui s'efforcent d'accéder à la manne. Il est difficile d'enrayer la dégradation en cours, mais cependant on tente depuis peu une campagne de protection en multipliant le gardiennage des églises. Ainsi, sur les instigations du bureau du tourisme et de la culture d'Ürgüp et le contrôle des autorités de Nevşehir, le monastère dit Hallaç manastiri près d'Ortahisar a été nettoyé et les graffiti effacés, et un gardien en contrôle les entrées grâce à un carnet à souche (le prix du ticket est modeste); de même à Saint-Théodore (IX ${ }^{\mathrm{e}}-\mathrm{X}^{\mathrm{e}}$ siècle), nommé Pancarlı kilise, où l'on a posé des grilles. Encore faudrait-il que soient poursuivies sévèrement ces mesures et qu'elles soient généralisées.

Mais le gardiennage n'est efficace que si les visiteurs sont en petit nombre. Si l'affluence est grande il faut des mesures d'un tout autre ordre.

La pollution touristique atteint fatalement les sites les plus visités et la Cappadoce n'est que l'un d'entre eux; Ephèse, Korikos, Hiérapolis, etc. témoignent de ses attaques. Elle n'est d'ailleurs pas particulière à la Turquie et a déjà donné lieu à des nombreuses études et séminaires traitant des dommages et des moyens de préservation à proposer; le dernier colloque en date s'est tenu à Florence en juin 1988. De plus en plus on envisage de fermer certains monuments aux touristes et de leur offrir des copies, comme à Lascaux. En Égypte, on obture les tombes que les touristes dégradent. En Cappadoce, certains muhtars ou propriétaires d'églises l'ont fait spontanément.

45. Des plans ont existé (cf. note 18) mais sont restés lettres mortes. 


\section{BIBLIOGRAPHIE}

ARTS DE CAPPADOCE, éd. Nagel, Genève-Paris-Munich 1971.

DOSSIERS-ARCHEOLOGIA n ${ }^{\circ}$ 63: La Cappadoce aux surprenantes richesses, Dossiers Histoire et Archéologie, $n^{\circ}$ 63, mai 1982.

DOSSIERS-ARCHEOLOGIA n ${ }^{\circ}$ 121: L'art religieux de Cappadoce, Dossiers Histoire et Archéologie, $\mathrm{n}^{\circ} 121$, novembre 1987.

EPSTEIN 1986: Ann WHARTON EPSTEIN, Tokalı kilise. Tenth-century Metropolitan Art in Byzantine Cappadocia, DOSt 22, Washington 1986.

EYICE: EYICE, S., Karadağ (Binbirkilise) ve Karaman, Istanbul 1971 (en turc, résumé français).

FIRATLI, N:: FIRATLI, N., "An Early Byzantine Hypogeum Discovered at Iznik», Mélanges Mansel, Ankara 1974, p. 919-32, pl. 327-40.

GABRIEL: GABRIEL, A., Voyages archéologiques dans la Turquie orientale, Paris 1940.

GÖREME HISTORICAL NATIONAL PARK. Master Plan for Protection and Use, s. 1. 1972.

GOUGH 1964 et 1965: GOUGH, M., "The Monastery of Eski Gümüş. Preliminary report / Second preliminary Report», AnatSt 14 (1964), p. 147-62; et 15 (1965), p. 157-64.

JERPHANION: G. de JERPHANION, Une nouvelle province de l'art byzantin. Les peintures rupestres de Cappadoce, Paris 1925-1942.

LABORDE: L. de LABORDE, Voyage en Orient, I, Asie Mineure, Paris 1837.

ÖTÜKEN 1987: ÖTÜKEN, Yıldiz, «Niğde'nin eski Andaval Köyündeki. Konstantinos Kilisesinin freskolari», Ayribasım, Ankara Universitesi, 1987, p. 125-45.

RAMSAY-BELL: RAMSAY, W. et Gertrude BELL, The Thousand and One Churches, London 1909.

RESTLE 1967: RESTLE, M., Die byzantinische Wandmalerei in Kleinasien, Recklinghausen 1967.

RESTLE 1979: RESTLE, M., Studien zur Frühbyzantinischen Architektur Kappadokiens, Wien 1979.

RESTLE 1986: RESTLE, M., "Zum Stil kleinasiatischer Wandmalereien in der 1. Hälfte des 13. Jarhunderts», Studenica et l'Art byzantin autour de l'année 1200, Belgrade 1988, p. 349-58.

RODLEY 1985: RODLEY, Lyn, Cavc monastcrics of Byzantinc Cappadocia, CambridgeLondon, 1985.

SCHIEMENZ 1986: SCHIEMENZ, G., «Die Doppelkirche von Babayan», Istanbuler Mitteilingen 36 (1986), p. 183-221.

THIERRY, N. et M., 1963: THIERRY, Nicole et Michel, Nouvelles églises rupestres de Cappadoce. Région du Hasan dağı, Paris 1963.

THIERRY, N., 1981: THIERRY, N., "À propos de la restauration des peintures murales", Terzo Simposio internazionale di Artè Armenia, Atti, Venezia 1981, p. 583-96.

THIERRY, N., 1983. Haut Moyen Age en Cappadoce. Les églises de la région de Çavuşin, I, Paris 1983. 